\title{
Primary care patients' expectations regarding medical appointments and their experiences during a visit: does age matter?
}

This article was published in the following Dove Press journal:

Patient Preference and Adherence

14 July 2017

Number of times this article has been viewed

\author{
Mariusz Jaworski' \\ Marta Rzadkiewicz' \\ Miroslawa Adamus' \\ Joanna Chylinska' \\ Magdalena Lazarewicz' \\ Gørill Haugan² \\ Monica Lillefjell ${ }^{3}$ \\ Geir Arild Espnes ${ }^{2}$ \\ Dorota Wlodarczyk' \\ 'Department of Medical Psychology, \\ Second Faculty of Medicine, Medical \\ University of Warsaw, Warsaw, \\ Poland; ${ }^{2}$ Department of Public \\ Health and Nursing, ${ }^{3}$ Department \\ of Neuromedicine and Movement \\ Science, NTNU Center for Health \\ Promotion Research, Norwegian \\ University of Science and \\ Technology, Trondheim, Norway
}

Correspondence: Mariusz Jaworski Department of Medical Psychology, Second Faculty of Medicine, Medical University of Warsaw, ZJAM Building-First Floor, 8I Zwirki i Wigury Street, Warsaw 02-09I, Poland

$\mathrm{Tel}+48225720533$

Fax +48 225720542

Email jaworskimariuszl@gmail.com
Introduction: There is evidence that meeting patients' expectations toward health care correlates with involvement in the treatment they receive. The most important patient expectations concern certain types of information: explanation of disease and treatment, health promotion, and improvement in quality of life. Other demands include proper rapport and emotional support. The aim of this paper was to examine different patient groups over the age of 50 years and their expectations toward medical visits, evaluated before a visit and after the visit.

Patients and methods: The study group consisted of 4,921 primary health-care patients. The patients received self-administered questionnaires that they filled in before and after the appointment with the doctor. Interviews with patients were conducted individually by specially trained interviewers. The PRACTA Patient Expectations Scale was used to measure the appointment-related expectations of the patients.

Results: We observed differences related to age in patients' expectations before medical visits regarding the following factors: disease explanation, treatment explanation, quality of life, rapport, and emotional support. The same differences were not observed on health promotion. Evaluation of patients' appointment-related experiences after the visit showed that there were significant differences between the age-groups regarding all types of expectations included in the study. Differences between previsit and postvisit measurements were statistically significant in all age-groups. Patients who received less than they expected from doctors outnumbered those who received what they expected or more in all the groups.

Conclusion: Patients' expectations toward medical visits are conditioned by age. Therefore, doctors should pay more attention to requirements related to age in their effort to identify and satisfy expectations. This is particularly important in light of the discrepancy between previsit expectations and the actual experiences of patients evaluated after the visit.

Keywords: primary care, patients expectations, medical appointment, experiences, PRACTA

\section{Introduction}

The aging of the population has caused an increase in the general number of elderly patients, including in primary health care. According to the World Health Organization (WHO), the number of people aged 65 years or older is expected to reach nearly 1.5 billion, $16 \%$ of the world's population, by 2050. As a result, many developed countries will have the oldest global population profiles, as well as rapidly aging populations. The WHO stresses that between 2010 and 2050, the number of people aged 65 years or older is projected to increase by more than $250 \%$ in less developed countries and $71 \%$ in developed countries. ${ }^{1}$ This leads to a serious social and medical challenge. To maintain the efficacy of medical care, high-quality programs promoting 
a healthy lifestyle and targeting older age-groups are urgently needed. ${ }^{1-3}$ According to the report, seniors should be encouraged by doctors to be more active in the treatment and health-promotion process. ${ }^{4,5}$ The effectiveness of such programs depends on the motivation of seniors to perform a given activity and their expectations toward health and medical care.

There is evidence that patients who receive medical care in line with their expectations may recover more quickly than patients who do not. Moreover, meeting the expectations of patients toward health care correlates with their satisfaction with medical visits. ${ }^{6-10}$ According to patients' expectations of health care, it is important to analyze patients' previsit expectations and postvisit experiences, as well as how they change according to health activity. ${ }^{7,11}$

However, some difficulties occur when attempting to define accurately what an expectation toward medical care is. . $^{7,12-14}$ Very often, the expectations of patients toward their interaction with health-care professionals are based on cognitive and affective beliefs. ${ }^{15,16}$ There are publications that analyze different dimensions of patients' expectations, such as structure of health care, doctor-patient communication style, the doctor's approach to sharing information, indicators of the quality of health care, and the patients' health outcomes..$^{7,8,14,17}$ The literature emphasizes that a fully integrated model of expectations should be developed in research projects. This model needs to be dynamic, multidimensional, and able to identify different factors, including sociodemographic ones. ${ }^{7}$

In the context of primary care, the most important patient expectations are associated with information about disease and treatment. ${ }^{18-21}$ When it comes to medical visits, patients have specific expectations of a clear diagnosis of their disease, explanations of the cause of their problem, and possible forms of therapy. ${ }^{7,8,18-21}$ In the literature, it is stressed that a patient, particularly those above the age of 65 years, tends to share their psychological and emotional problems with a family doctor. As a result, the emotional support provided to especially elder patients is extremely important. ${ }^{22}$ Another key aspect of a medical visit is the relationship between the patient and the doctor, as they form a mutual bond. It is emphasized that building a successful rapport is the single most important factor in developing a relationship. In the same vein, BakićMirić and Bakić ${ }^{23}$ pointed out that building a successful rapport largely depends on the validity of the patient expectations and the ability of the doctor to fulfill them. It should be noted that within complementary therapy, patients also value enhanced quality of life and health promotion. ${ }^{24-26}$
Overall, patients visiting GPs report not only high previsit expectations but also that the expectations are fulfilled. These expectations vary according to patient category. For example, patients seeing GPs have higher previsit expectations than hospital patients, and their postvisit ratings of how their expectations were satisfied are higher. ${ }^{7}$ It seems that patients' expectations may depend on sociodemographic variables. For example, Strzelecka et $\mathrm{al}^{27}$ stressed that the declared patients' expectations vary according to age-group, especially with regard to emotional support and obtaining information about a given disease and treatment. Similar observations were reported by Kemicer-Chmielewska et al. ${ }^{22}$ Therefore, an important activity in the field of public health is to study patients in terms of their psychological, health and sociodemographic variables.

In short, according to previous studies, the most important patient expectations concern information about disease and treatment, quality of life, health promotion, rapport, and emotional support. ${ }^{18-26}$ However, it should be said that these expectations tend to be analyzed separately. There is a scarcity of studies analyzing various types of patients' expectations together and presenting a comprehensive picture of them. What is more, patients' expectations have been examined more closely than their experiences, and little is known about the relationship between expectations and actual experiences, especially among older adults in the primary health-care services. In order to respond to this, we have here adopted a comprehensive and multidimensional approach to patients' expectations and focused on both patients' expectations and experiences during a medical appointment. We used this approach in relation to older patients within the primary health-care services, as the knowledge about this group and the aforementioned aspects is still insufficient. The approach was used in the Polish-Norwegian PRACTA study of patients aged 50 or more, who were examined both before and after visiting a doctor in the primary healthcare services.

\section{Aims}

One of the most important objectives of the PRACTA study was to examine patients' expectations toward their consultation with a primary health-care physician. This paper aims at studying appointment-related expectations before a visit, the actual experiences after the visit, and the discrepancy between expectations and experiences in the different agegroups. Three research questions were formulated:

1. Do patients differ in appointment-related expectations before a visit according to their age? 
2. Do the experiences of the patients evaluated after a visit differ according to patient age?

3. What is the relationship between the appointment-related expectations (previsit) and the actual experience evaluated after the visit (postvisit) for the various age-groups?

\section{Patients and methods Procedure and general description of study sample}

The study group consisted of 4,921 primary health-care patients; 1,595 patients declined to participate $(52.6 \%$ of women and $47.4 \%$ of men). Further information about this group was not collected. The age of the patients ranged from 50 to 98 (mean 68.9, SD 9.1) years. In the next stage of the analysis, the group was divided into four age categories: 1) the youngest group consisting of 1,529 respondents aged 50-64 (mean 58.24, SD 4.35) years, 2) the "young-old" group consisting of 2,011 respondents aged 65-74 (mean 69.29, SD 2.75) years, 3 ) the "middle-old" group consisting of 1,180 respondents aged $75-84$ (mean 78.67, SD 2.68) years, and 4) the "old-old" group consisting of 201 respondents aged above 85 (mean 87.58, SD 2.59) years.

Inclusion criteria were aged $>50$ years, able to complete questionnaires independently, awaiting a visit to a doctor recruited for the PRACTA study, and written consent to participate. The participants were given all relevant information about the study's background, purpose, design, procedure, voluntary nature of participation, and possibility to withdraw at any time. The study was approved by the Bioethics Committee of the Medical University of Warsaw (KB/10/2014). The patients filled in questionnaires before and immediately after the appointment with the doctor. Interviews with patients were carried out individually by specially trained interviewers.

\section{Socioeconomic characteristics of respondents}

The socioeconomic characteristics of the respondents in the various age-groups are presented in Table 1. The four groups were not homogeneous in terms of sex, and contained people with different marital status, with the number of widowed seniors increasing with age. In the four groups analyzed, the clear majority were women. However, women were not dominant in the older groups. Probably, this was due to the fact that women were generally less willing to participate.

There were also differences in education. With increasing age, patients with primary education were more numerous, whereas the number of respondents with a secondary or higher education decreased. The respondents' place of residence was not equally balanced, as patients who lived in a large town or city dominated in each age-group (Table 1).

There were also differences between the groups when various categories of flat-sharing were analyzed. The number of participants living alone varied according to age-group. Most patients who lived alone (40.3\%) were in the middle-old group. The groups further differed according to the number who lived with a spouse or partner, children, grandchildren, or non-family members. With higher age, the percentages of patients living with a spouse or partner dropped. When it came to patients living with family members, there were no differences observed between the analyzed age-groups. Most respondents who lived with family members were in the group of patients aged $85+$ years (Table 1 ). The groups also differed also with regard to occupational status. The number of patients with full-time or part-time employment and those unemployed decreased with lower age. The number of retired patients predictably increased with higher age. There were differences in the number of pensioners, with the lowest percentage observed in the middle-old group (11\%) (Table 1). When it came to financial status, the number of people with poor or average income increased with higher age (Table 1).

\section{Health status of participants}

The health-status characteristics of the sample included the following factors: subjective health status, self-reported number of diseases, use of health care within the past 6 months, and aim of the current visit. Results regarding the health status of the various age-groups are presented in Table 2. The groups differed according to their subjective health status. The number of patients declaring good health decreased with higher age, whereas patients declaring poor health increased. We found similar differences in the self-reported number of diseases, which increased with higher age. The nature of hospital care used in the analyzed age-groups was different. With increasing age, a higher frequency of emergency care was observed. There were also differences in hospital admissions for surgery or other procedures. Patients aged 50-64 years reported the lowest percentage of such admissions (8.5\%). Admission for treatment or observation was most common among patients aged 75-84 years. The use of medical care, such as visits to specialists, was less common among patients aged 50-64 years than for the other groups. The use of medical tests was more evident among patients aged 75-84 years, though other types of visits to GPs were more common among patients aged 65-75 years. No differences were observed with regard to 
Table I Socioeconomic profile of respondents

\begin{tabular}{|c|c|c|c|c|c|c|}
\hline & $\begin{array}{l}50-64 \\
\text { years old } \\
n=1,529 \\
\end{array}$ & $\begin{array}{l}\text { "Young old" } \\
\text { (65-74 years) } \\
n=2,01 \text { I } \\
\end{array}$ & $\begin{array}{l}\text { “Middle old" } \\
\text { (75-84 years) } \\
n=I, 180 \\
\end{array}$ & $\begin{array}{l}\text { “Old old” } \\
\text { (85+ years) } \\
n=201 \\
\end{array}$ & $x^{2}$ & Cramér's $\varphi$ \\
\hline & n (\%) & n (\%) & n (\%) & n (\%) & & \\
\hline \multicolumn{7}{|l|}{ Sex } \\
\hline Female & $847(55.4)$ & $\mathrm{I}, 176(58.5)$ & $723(6 \mid .3)$ & $112(55.7)$ & $10.05^{*}$ & $0.05^{*}$ \\
\hline Male & $682(44.6)$ & $835(41.5)$ & $457(36.7)$ & $89(44.3)$ & & \\
\hline \multicolumn{7}{|l|}{ Marital status } \\
\hline Single & $82(5.4)$ & $103(5.1)$ & $46(3.9)$ & $18(9)$ & $617.58 *$ & $0.35^{*}$ \\
\hline Married/partnered & $\mathrm{I}, 122(73.4)$ & $1,210(60.2)$ & $458(38.8)$ & $69(34.3)$ & & \\
\hline Divorced/separated & $145(9.5)$ & $144(7.2)$ & $46(3.9)$ & $12(6)$ & & \\
\hline Widowed & $180(11.8)$ & $554(27.5)$ & $630(53.4)$ & $102(50.7)$ & & \\
\hline \multicolumn{7}{|l|}{ Education level } \\
\hline Primary & $47(3.1)$ & I55 (7.7) & $220(18.6)$ & $53(26.4)$ & $445.02 *$ & $0.3^{*}$ \\
\hline Vocational & $373(24.4)$ & $679(33.8)$ & $440(37.3)$ & $76(37.8)$ & & \\
\hline Secondary & $748(48.9)$ & $868(43.2)$ & $4,120(34.7)$ & $58(28.9)$ & & \\
\hline Higher & $361(23.6)$ & $309(15.4)$ & $110(9.3)$ & $14(7)$ & & \\
\hline \multicolumn{7}{|l|}{ Place of residence } \\
\hline Rural area & I8I (1।.8) & $168(8.4)$ & $127(10.8)$ & $29(14.4)$ & $115.2^{*}$ & $0.15^{*}$ \\
\hline Small town & $135(8.8)$ & $90(4.5)$ & $82(6.9)$ & $9(4.5)$ & & \\
\hline Medium town & $220(14.4)$ & $243(12.1)$ & $146(12.6)$ & $14(7)$ & & \\
\hline Large town & $993(64.9)$ & $1,510(75.1)$ & $825(69.9)$ & $149(74.1)$ & & \\
\hline \multicolumn{7}{|c|}{ Who do you live with? (yes/no) } \\
\hline Alone & $24 \mid(15.8)$ & $530(26.4)$ & $475(40.3)$ & $51(25.4)$ & $205.94 *$ & $0.21 *$ \\
\hline Spouse/partner & $\mathrm{I}, 12 \mathrm{I}(73.3)$ & $1,220(60.7)$ & 457 (38.7) & $67(33.3)$ & $383.67^{*}$ & $0.28 *$ \\
\hline Children & $531(34.7)$ & $385(19.1)$ & $283(24)$ & $61(30.3)$ & I $14.88^{*}$ & $0.15^{*}$ \\
\hline Grandchildren & $76(5)$ & $153(7.6)$ & $148(12.5)$ & $30(14.9)$ & $63.23 *$ & $0.11 *$ \\
\hline Other family members & $68(4.4)$ & $90(4.5)$ & $57(4.8)$ & $16(8)$ & 5.27 & - \\
\hline Non-family members & $36(2.4)$ & $25(1.2)$ & $12(\mathrm{I})$ & $5(2.5)$ & $10.8^{* *}$ & $0.05^{*}$ \\
\hline Nursing home & $4(0.3)$ & $4(0.2)$ & $6(0.5)$ & $2(1)$ & 5.19 & - \\
\hline \multicolumn{7}{|l|}{ Work status } \\
\hline Full-time & $657(43)$ & $|2|(6)$ & $8(0.7)$ & $4(2)$ & $\mathrm{I}, 208.76 *$ & $0.5^{*}$ \\
\hline Part-time & $202(13.2)$ & $185(9.2)$ & $18(1.5)$ & $3(1.5)$ & $134.12 *$ & $0.17^{*}$ \\
\hline Retired & $398(26)$ & I,523 (75.7) & $\mathrm{I}, 027(87)$ & $176(87.6)$ & I,387.96* & $0.53^{*}$ \\
\hline Pensioner & $298(19.5)$ & $260(12.9)$ & $130(\mathrm{II})$ & $26(12.9)$ & $46.63 *$ & $0.1 *$ \\
\hline Unemployed & $76(5)$ & $17(0.8)$ & $17(1.4)$ & 0 & $78.13^{*}$ & $0.13^{*}$ \\
\hline \multicolumn{7}{|l|}{ Financial situation } \\
\hline Poor & $26(1.7)$ & $64(3.2)$ & $34(2.9)$ & $8(4)$ & $84.00 *$ & $0.13^{*}$ \\
\hline Rather poor & $208(13.6)$ & $262(22.2)$ & $262(22.2)$ & $23(11.4)$ & & \\
\hline Average & $865(56.6)$ & $669(56.7)$ & $669(56.7)$ & $132(65.7)$ & & \\
\hline Rather good & $361(23.6)$ & $174(14.7)$ & $174(14.7)$ & $29(14.4)$ & & \\
\hline Good & $69(4.5)$ & $4 \mid(3.5)$ & $4 \mid(3.5)$ & $9(4.5)$ & & \\
\hline
\end{tabular}

Notes: $* P<0.01 ; * * P<0.05$

sanatorium admissions. The analyzed groups differed with regard to the aims of the visit ( $\chi^{2}=22.78, P<0.01$; Cramér's $\varphi=0.04 ; P<0.01)$. In approximately $10 \%$ of visits, patients received referrals (eg, to a specialist or for tests) rather than direct medical help. Referrals were less common than appointments concerning medical help (treatment, checkup, diagnosis) in all groups. However, patients aged 50-64 years and old-old patients reported formal reasons for appointments more often. Patients aged 75-84 years more often than the other groups declared that their visits concerned medical help (treatment, checkup, diagnosis).

\section{Questionnaires}

The PRACTA Patient Expectations Scale was used to measure a patient's appointment-related expectations. The scale has two versions containing identical sets of 18 items, but with different statements. The previsit version refers to patients' expectations toward the doctor before the visit ("During this visit, it is important to me that the doctor ..."), whereas the postvisit version refers to the doctor's action during the visit, as evaluated after a visit ("During this visit, the doctor ..."). Each item has a 7-point response range (1, definitely not; 7, definitely so). 
Table 2 Health status of participants

\begin{tabular}{|c|c|c|c|c|c|c|}
\hline & $\begin{array}{l}50-64 \\
\text { years old } \\
n=1,529 \\
n(\%)\end{array}$ & $\begin{array}{l}\text { "Young old" } \\
\text { (65-74 years) } \\
n=2,011 \\
n(\%)\end{array}$ & $\begin{array}{l}\text { “Middle old" } \\
\text { (75-84 years) } \\
\mathrm{n}=1,180 \\
\mathrm{n}(\%)\end{array}$ & $\begin{array}{l}\text { "Old old" } \\
\text { (85+ years) } \\
n=201 \\
n(\%)\end{array}$ & $\chi^{2}$ & $\begin{array}{l}\text { Effect } \\
\text { size }\end{array}$ \\
\hline \multicolumn{7}{|c|}{ How do you evaluate your health (in comparison with people of a similar age)? } \\
\hline Very good & $35(2.3)$ & $37(1.8)$ & $22(1.9)$ & $3(1.5)$ & $175.14 * *$ & $0.03^{\mathrm{a}}$ \\
\hline Good & $497(32.5)$ & $373(18.5)$ & $191(16.2)$ & $27(13.4)$ & & \\
\hline Average & $811(53)$ & $1,255(62.4)$ & $718(60.8)$ & $90(44.8)$ & & \\
\hline Poor & I75 (I I.4) & $307(15.3)$ & $223(18.9)$ & $75(37.3)$ & & \\
\hline Very poor & $\mathrm{II}(0.7)$ & $39(1.9)$ & $26(2.2)$ & $6(3)$ & & \\
\hline \multicolumn{7}{|c|}{ How many diseases have you had/are you currently being treated for? } \\
\hline None & $383(25)$ & $158(7.9)$ & $66(5.6)$ & $6(3)$ & $251.6 I^{* *}$ & $0.05^{\mathrm{a}}$ \\
\hline I disease & $45 \mathrm{I}(29.5)$ & $686(34.1)$ & $325(27.5)$ & $45(22.4)$ & & \\
\hline $2-3$ diseases & $485(31.7)$ & $804(40)$ & $497(42.1)$ & $77(38.3)$ & & \\
\hline $4-5$ diseases & $170(\mid 1.1)$ & $29 \mid(\mid 4.5)$ & $235(19.9)$ & $45(22.4)$ & & \\
\hline 6 or more diseases & $40(2.6)$ & $72(3.6)$ & $57(4.85)$ & $28(13.9)$ & & \\
\hline \multicolumn{7}{|c|}{ Have you attended the following within the past 6 months?\# } \\
\hline Emergency room & $32(2.1)$ & $82(4.1)$ & $49(4.2)$ & $13(6.5)$ & $17.23 * *$ & $0.06 * *, b$ \\
\hline Surgery or other procedure in hospital & $130(8.5)$ & $253(12.6)$ & $157(13.3)$ & $21(10.4)$ & $19.91 * *$ & $0.01 * *, b$ \\
\hline Treated or observed in hospital & $53(3.5)$ & $120(6)$ & $90(7.6)$ & $9(4.5)$ & $23.57 * *$ & $0.07 * *, b$ \\
\hline Visited other general practitioner & $388(25.4)$ & $637(31.7)$ & $292(24.7)$ & $57(28.4)$ & $24.94 * *$ & $0.07 * *, b$ \\
\hline Visited a specialist & $634(4 I .5)$ & $\mathrm{I}, \mathrm{IOI}(54.7)$ & $698(59.2)$ & $89(44.3)$ & $101.85^{* *}$ & $0.14 * *, \mathrm{~b}$ \\
\hline $\begin{array}{l}\text { General medical tests (blood count, } \\
\text { electrocardiogram, etc) }\end{array}$ & $586(38.3)$ & $878(43.7)$ & $593(50.3)$ & $77(38.3)$ & $40.78 * *$ & $0.09 * *, b$ \\
\hline Sanatorium & $146(9.5)$ & $157(7.8)$ & $83(7)$ & $13(6.5)$ & 7.06 & - \\
\hline
\end{tabular}

Notes: ${ }^{* * P}<0.05$; a $\eta^{2}$; ${ }^{\circ}$ Cramér's $\varphi$; ${ }^{\#}$ response options yes/no.

Each version of the scale consists of six three-item thematic sections involving expectations toward the following factors:

1. Disease explanation (Cronbach's $\alpha=0.914$ and $\alpha=0.893$ for pre and postvisit versions, respectively), eg, "The doctor explains/explained the possible consequences of the illness"

2. Treatment explanation (Cronbach's $\alpha=0.829$ and $\alpha=0.809$ for pre- and postvisit versions, respectively), eg, "The doctor presents/presented recommendations for the treatment"

3. Emotional support (Cronbach's $\alpha=0.911$ and $\alpha=0.897$ for pre- and postvisit versions, respectively), eg, "The doctor shows/showed me that s/he cares"

4. Health promotion (Cronbach's $\alpha=0.901$ and $\alpha=0.885$ for pre- and postvisit versions, respectively), eg, "The doctor suggests/suggested what I can do to improve how I function in everyday life"

5. Quality-of-life improvement (Cronbach's $\alpha=0.951$ and $\alpha=0.942$ for pre- and postvisit versions, respectively), eg, "The doctor suggests/suggested ways of maintaining life satisfaction"

6. Rapport (Cronbach's $\alpha=0.896$ and $\alpha=0.925$ for pre- and postvisit versions, respectively), eg, "The doctor treats/ treated me seriously".
A three-part survey was used to identify patient characteristics. The first part concerned sociodemographic factors: age, sex, marital status, education, place of residence, flatsharing, professional, and financial status. Part two contained questions about health, including self-rated health ("How do you evaluate your health in comparison with people of similar age?"), use of the health-care system within the past 6 months ("Within the past 6 months, have you used emergency services, undergone surgery or other procedures in hospital, been treated or observed in a hospital, visited other general practitioners, visited a specialist, undergone general medical tests [blood count, ECG, etc], or been in a sanatorium?" [multiple choice]), and number of current diseases ("How many diseases have you been treated for/are you currently being treated for?"). The last part of the survey concerned the aim of the medical visit, with multiple-choice questions.

\section{Statistical analysis}

SPSS version 21 was used (IBM Corporation, Armonk, NY, USA). In all parts of the analysis, the default level of significance was set at $P=0.05$. As the outcome variables were not normally distributed, nonparametric statistical tests were applied. To assess between-group differences, $\chi^{2}$, Mann-Whitney $U$, or Kruskal-Wallis tests were used. The effect size was calculated for statistically 
significant differences. In these cases, Cramér's $\varphi$ and $\eta^{2}$ statistics were used. Differences in previsit and postvisit measurements were examined by the Wilcoxon signed-rank test.

A discrepancy index was used to indicate the difference between postvisit and previsit measurements. An index value below zero meant that the patient received less than he/she had expected from the GP before the visit. The KruskalWallis test was used to determine between-group differences. Next, we carried out post hoc multiple comparisons for statistically significant differences between the analyzed groups using Dunn's test.

\section{Results}

\section{Age and appointment-related expectations: previsit measurement}

As presented in Table 3, the age-groups differed in all types of expectations, except for health promotion. More specifically, patients aged 50-64 years scored higher in expectations toward disease explanation compared to participants aged 65-74 and 75-84 years. A very similar observation was made in the case of treatment explanation. Here, patients aged 50-64 years scored higher than participants aged 65-74 and 75-84 years. However, patients aged $85+$ years expected significantly more emotional support than patients aged 65-74 years. Expectations toward good rapport were higher among patients aged 50-64 years than patients aged 65-74 years. Interestingly, among patients aged 75-84 years, expectations toward quality of life were higher than among patients aged 50-64 years (Table 3). The mean levels of each expectation before the medical visit are shown in Table 4.

\section{Age and appointment-related experiences: postvisit measurement}

Evaluation of patients' appointment-related experiences evaluated after the visit showed that there were significant variations among the age-groups in all categories: disease explanation, treatment explanation, emotional support, health promotion, quality of life, and rapport (Table 5). The youngest patients (50-64 years) perceived that the doctor had less focus on disease explanation and treatment explanation than those aged $65-74$ and 75-84 years. No differences were evident between the youngest and old-old participants regarding these two dimensions. Patients aged 50-64 years indicated that the doctor had less emphasis on emotional support than patients aged 65-74, 75-84, and $85+$ years. The same pattern was observed with regard to health promotion and quality of life. It should be noted that patients aged $85+$ years reported a higher stress on rapport than those in the age-groups 50-64, 65-74, and 75-84 years. Patients aged 65-74 years also had higher scores on this dimension than those aged 75-84 years. Mean levels of patients' appointment-related experiences assessed after the visit are presented in Table 6 .

Table 3 Patients' previsit appointment-related expectations in selected age-groups

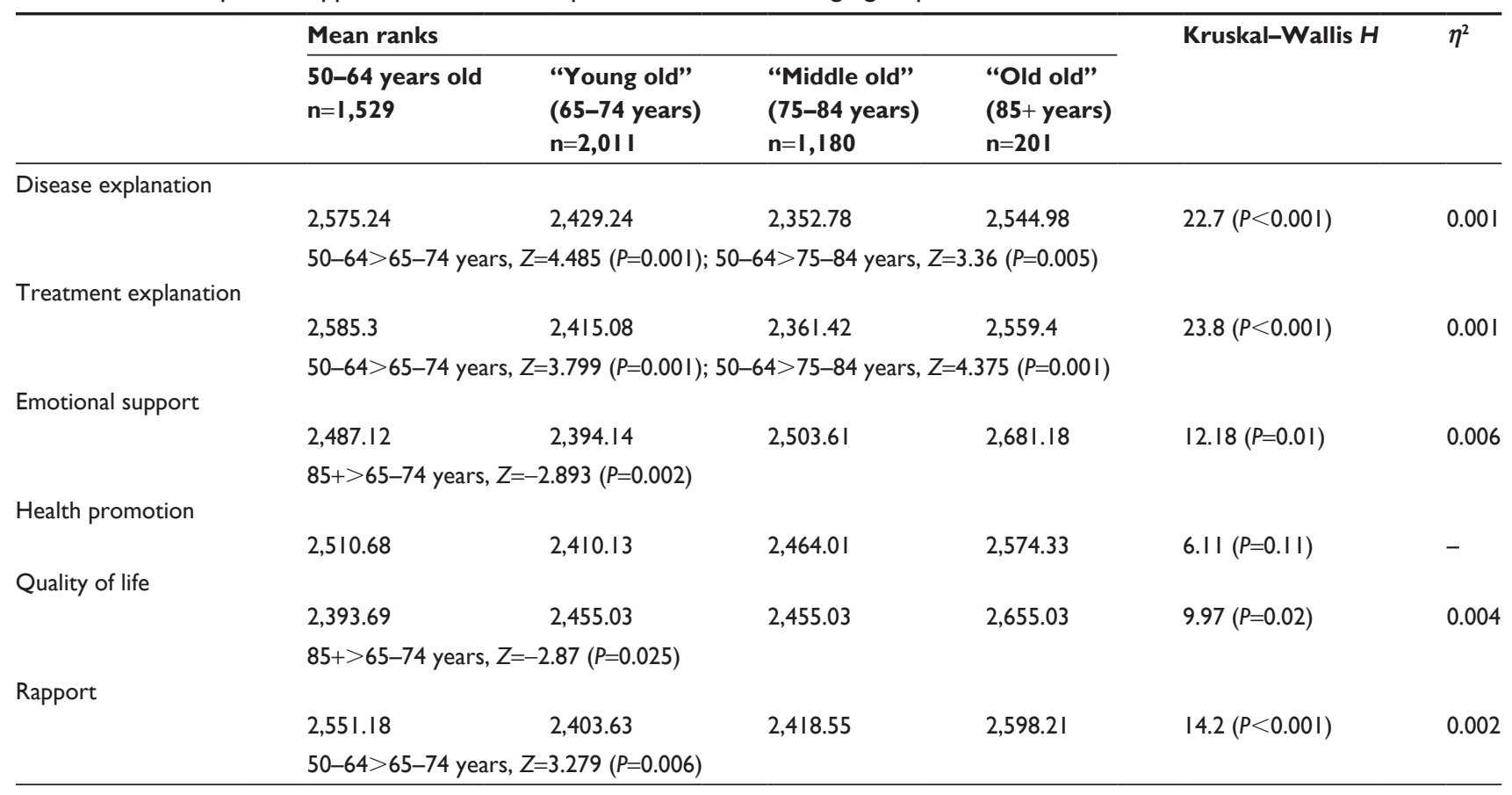


Table 4 Previsit patient appointment-related expectations

\begin{tabular}{|c|c|c|c|c|c|c|c|}
\hline \multicolumn{2}{|c|}{$\begin{array}{l}50-64 \text { years } \\
\text { old } \\
n=I, 529\end{array}$} & \multicolumn{2}{|c|}{$\begin{array}{l}\text { “Young old" } \\
\text { (65-74 } \\
\text { years) } \\
n=2,01 \text { I }\end{array}$} & \multicolumn{2}{|c|}{$\begin{array}{l}\text { “Middle old” } \\
\text { (75-84 } \\
\text { years) } \\
\mathrm{n}=I, 180 \\
\end{array}$} & \multicolumn{2}{|c|}{$\begin{array}{l}\text { "Old old" } \\
\text { (85+ years) } \\
n=201\end{array}$} \\
\hline Mean & SD & Mean & SD & Mean & SD & Mean & SD \\
\hline \multicolumn{8}{|c|}{ Disease explanation } \\
\hline 6.51 & 0.94 & 6.51 & 0.8 & 6.48 & 0.8 & 6.57 & 0.72 \\
\hline \multicolumn{8}{|c|}{ Treatment explanation } \\
\hline 6.43 & 0.94 & 6.41 & 0.83 & 6.4 & 0.77 & 6.51 & 0.67 \\
\hline \multicolumn{8}{|c|}{ Emotional support } \\
\hline 6.17 & 1.28 & 6.19 & 1.12 & 6.35 & 0.82 & 6.41 & 0.92 \\
\hline \multicolumn{8}{|c|}{ Health promotion } \\
\hline 6.09 & 1.27 & 6.1 & 1.13 & 6.2 & 0.93 & 6.24 & 0.98 \\
\hline \multicolumn{8}{|c|}{ Quality of life } \\
\hline 5.17 & 1.96 & 5.33 & 1.83 & 5.45 & I.7I & 5.53 & 1.77 \\
\hline \multicolumn{8}{|c|}{ Rapport } \\
\hline 6.47 & 0.7 & 6.4 & 0.72 & 6.42 & 0.69 & 6.48 & 0.73 \\
\hline
\end{tabular}

Notes: Data presented as a mean for each subscale. Each item has a 7-point response range (I, definitely not; 7 , definitely so).

\section{Differences between appointment-related expectations evaluated before visit and experiences evaluated after visit}

The results presented in Table 7 show that differences between previsit and postvisit measurements were statistically significant in all age-groups with regard to all types of expectations. In all age-groups, patients who received less than they expected on disease explanation, treatment explanation, emotional support, health promotion, quality of life, and rapport outnumbered those who got what they expected or more than they expected. It is also important to look at the proportions of patients who received what they expected or more from doctors (satisfied needs) and those who got less than they expected (neglected needs).

For patients aged 50-64 years, the highest number of those who received less than they expected (most neglected needs) received less in relation to emotional support (76\%) and health promotion (75\%), followed by disease explanation (73\%), treatment explanation and quality of life (both $72 \%$ ), and rapport (63\%). In the young-old group, most of those who received less than they expected indicated emotional support and health promotion (both 72\%), followed by disease and treatment explanation (both 67\%), quality of life (66\%), and rapport (63\%). In the middle-old group, the highest percentages of those who received less than they expected indicated health promotion (76\%) and emotional support (75\%), followed by quality of life (71\%), rapport (68\%), treatment explanation (66\%), and disease explanation (65\%). In the old-old group, the highest number of those who felt they had received less than they expected mentioned treatment

Table 5 Patients' appointment-related experiences evaluated after a visit in selected age-groups

\begin{tabular}{|c|c|c|c|c|c|c|}
\hline & \multicolumn{4}{|l|}{ Mean ranks } & \multirow[t]{2}{*}{ Kruskal-Wallis $H$} & \multirow[t]{2}{*}{$\eta^{2}$} \\
\hline & $\begin{array}{l}50-64 \text { years old } \\
n=I, 529\end{array}$ & $\begin{array}{l}\text { “Young old" } \\
\text { (65-74 years) } \\
\text { n=2,0 I I }\end{array}$ & $\begin{array}{l}\text { “Middle old" } \\
\text { (75-84 years) } \\
n=I, 180\end{array}$ & $\begin{array}{l}\text { "Old old" } \\
\text { (85+ years) } \\
n=201\end{array}$ & & \\
\hline \multicolumn{7}{|l|}{ Disease explanation } \\
\hline & $2,271.01$ & $2,560.91$ & $2,560.76$ & 2,321 & $45.75(P<0.001)$ & 0.015 \\
\hline & \multicolumn{6}{|c|}{$50-64<65-74$ years, $Z=-6.06(P=0.00 I) ; 50-64<75-84$ years, $Z=-5.3(P=0.00 I)$} \\
\hline \multicolumn{7}{|l|}{ Treatment explanation } \\
\hline & $2,266.19$ & $2,586.39$ & $2,507.25$ & $2,416.91$ & $46.42(P<0.001)$ & 0.014 \\
\hline & \multicolumn{6}{|c|}{$50-64<65-74$ years, $Z=-6.68(P=0.00 \mathrm{I}) ; 50-64<75-84$ years, $Z=-4.4 \mathrm{I}(P=0.00 \mathrm{I})$} \\
\hline \multicolumn{7}{|l|}{ Emotional support } \\
\hline & $2,178.79$ & $2,618.5$ & $2,533.76$ & $2,604.87$ & $91.27(P<0.001)$ & 0.022 \\
\hline & \multicolumn{6}{|c|}{$50-64<65-74$ years, $Z=-6.68(P=0.00 I) ; 50-64<75-84$ years, $Z=-4.4 I(P=0.00 I) ; 50-64<85+$ years, $Z=-9.18(P=0.00 I)$} \\
\hline \multicolumn{7}{|l|}{ Health promotion } \\
\hline & $2,224.75$ & $2,598.45$ & $2,500.56$ & $2,650.73$ & $66.71(P<0.001)$ & 0.016 \\
\hline & \multicolumn{6}{|c|}{$50-64<65-74$ years, $Z=-7.82(P=0.00 \mathrm{I}) ; 50-64<75-84$ years, $Z=-5.05(P=0.00 \mathrm{I}) ; 50-64<85+$ years, $Z=-4.03(P=0.00 \mathrm{I})$} \\
\hline \multicolumn{7}{|l|}{ Quality of life } \\
\hline & $2,087.23$ & $2,658.28$ & $2,597.83$ & $2,527.2$ & $157.25(P<0.001)$ & 0.033 \\
\hline & \multicolumn{6}{|c|}{$\begin{array}{l}50-64<65-74 \text { years, } Z=-11.89(P=0.00 I) ; 50-64<75-84 \text { years, } Z=-9.31(P=0.00 I) ; 50-64<85+\text { years, } Z=-4.14 \\
(P=0.00 I)\end{array}$} \\
\hline \multicolumn{7}{|l|}{ Rapport } \\
\hline & $2,455.42$ & $2,511.23$ & $2,319.17$ & $2,833.55$ & $28.7(P<0.001)$ & 0.005 \\
\hline & \multicolumn{6}{|c|}{$\begin{array}{l}50-64<65-74 \text { years, } Z=-3.58(P=0.002) ; 65-74<85+\text { years, } Z=-3 . I(P=0.012) ; 75-84<85+\text { years, } Z=-4.79(P=0.00 I) ; \\
65-74>75-84 \text { years, } Z=3.72(P=0.00 I)\end{array}$} \\
\hline
\end{tabular}


Table 6 Patient appointment-related experiences evaluated after a visit

\begin{tabular}{|c|c|c|c|c|c|c|c|}
\hline \multicolumn{2}{|c|}{$\begin{array}{l}\text { 50-64 years } \\
\text { old } \\
n=I, 529\end{array}$} & \multicolumn{2}{|c|}{$\begin{array}{l}\text { “Young old" } \\
\text { (65-74 } \\
\text { years) } \\
n=2,01 \text { I }\end{array}$} & \multicolumn{2}{|c|}{$\begin{array}{l}\text { “Middle old" } \\
\text { (75-84 } \\
\text { years) } \\
n=I, 180\end{array}$} & \multicolumn{2}{|c|}{$\begin{array}{l}\text { "Oldest old" } \\
\text { (85+ years) } \\
n=20 \text { I }\end{array}$} \\
\hline Mean & SD & Mean & SD & Mean & SD & Mean & SD \\
\hline \multicolumn{8}{|c|}{ Disease explanation } \\
\hline 5.19 & $\mathrm{I} .42$ & 5.51 & 1.17 & 5.52 & 1.15 & 5.34 & 1.08 \\
\hline \multicolumn{8}{|c|}{ Treatment explanation } \\
\hline 5.05 & 1.49 & 5.41 & 1.27 & 5.37 & 1.21 & 5.31 & 1.05 \\
\hline \multicolumn{8}{|c|}{ Emotional support } \\
\hline 4.67 & 1.37 & 5.08 & 1.21 & 5.02 & 1.17 & 5.14 & 1.11 \\
\hline \multicolumn{8}{|c|}{ Health promotion } \\
\hline 4.7 & 1.41 & 5.05 & 1.23 & 5.01 & 1.14 & 5.16 & 1.08 \\
\hline \multicolumn{8}{|c|}{ Quality of life } \\
\hline 3.4 & 1.85 & 4.14 & 1.78 & 4.07 & 1.14 & 3.96 & 1.83 \\
\hline \multicolumn{8}{|c|}{ Rapport } \\
\hline 5.52 & 1.16 & 5.58 & 1.08 & 5.44 & 1.06 & 5.81 & 1.01 \\
\hline
\end{tabular}

explanation (75\%), followed by disease explanation and emotional support (both 74\%), health promotion $(73 \%)$, quality of life (66\%), and rapport (60\%). Significantly, in all age-groups the largest numbers of those who received exactly what they expected cited rapport, followed by disease and treatment explanation. Comparisons between the levels of discrepancy between pre-visit expectations and experiences evaluated after the visit are presented in Table 8 .

As shown in Table 8, the largest discrepancies between previsit expectations and experiences evaluated after the visit were evident in the youngest group, regardless of the type of expectation. This group differed from the young-old group in all expectations and from the middle-old group in five of six expectations (expect for rapport). In the case of the middle-old group, the lowest discrepancies were with regard to disease explanation. The young-old group was characterized by the lowest discrepancies in terms of treatment explanation, emotional support, health promotion, and quality of life. However, the lowest discrepancies on rapport were observed in the old-old group. Moreover, patients aged $85+$ years had a statistically higher difference between previsit expectations and experiences evaluated after the visit on disease explanation compared to patients aged 75-84 years. In the case of treatment explanation, patients aged 50-64 years had a statistically higher difference between previsit expectations and experiences evaluated after the visit than the young-old and the middle-old groups. A similar trend was observed with respect to emotional support and quality of life. Furthermore, when it came to expectations toward health promotion, the discrepancy between previsit measurements and postvisit measurements among the youngest patients was also higher than in the old-old group. In the case of rapport, patients aged $85+$ years had a statistically lower difference between previsit expectations and experiences evaluated after the visit than patients aged 50-64 years and those in the middle-old group.

\section{Discussion}

This study has highlighted patients' appointment-related expectations before a visit, their actual experiences evaluated after the visit, and the discrepancy between expectations and experiences among different age-groups. Differences in patients' expectations before medical visits were evident with regard to disease explanation, treatment explanation, quality of life, rapport, and emotional support. Our study showed that patients aged 50-64 years scored higher on expectations toward disease and treatment explanation before medical visits compared to the young-old and middle-old participants. Additionally, patients aged 50-64 years had higher rapport expectations than patients aged 65-74 years.

The youngest patients had the highest expectations in most categories, while the second-youngest age-group had the lowest expectations. This may be explained by less contact with medical services for patients who had not reached retirement age (50-65 years). Even if numerous diseases begin to occur at this age, the lack of experience with treatment may explain unrealistic expectations. Patients just below retirement age (often part time employees) or those who have just retired could have more time available to care for themselves. Subsequently, they might have more realistic expectations than younger people. One can also observe that differences among the age-groups are relatively small, and only on a few dimensions are there major differences between the youngest and oldest age-groups. This raises the question of whether patients' expectations evolve slowly instead of changing abruptly.

More pronounced differences were evident between the middle-old and old-old groups. In the old-old group, the highest numbers of patients who received less than they expected mentioned treatment and disease explanation, whereas in the middle-old group these expectations were best satisfied. Based on the mentioned percentages, we discern similar patterns for the youngest and young-old patients, but with the lowest number of patients receiving less than they expected in the young-old group.

Patients aged $85+$ years scored higher on emotional support and quality-of-life improvement compared to patients aged 65-74 years. Interestingly, the expectations of patients 


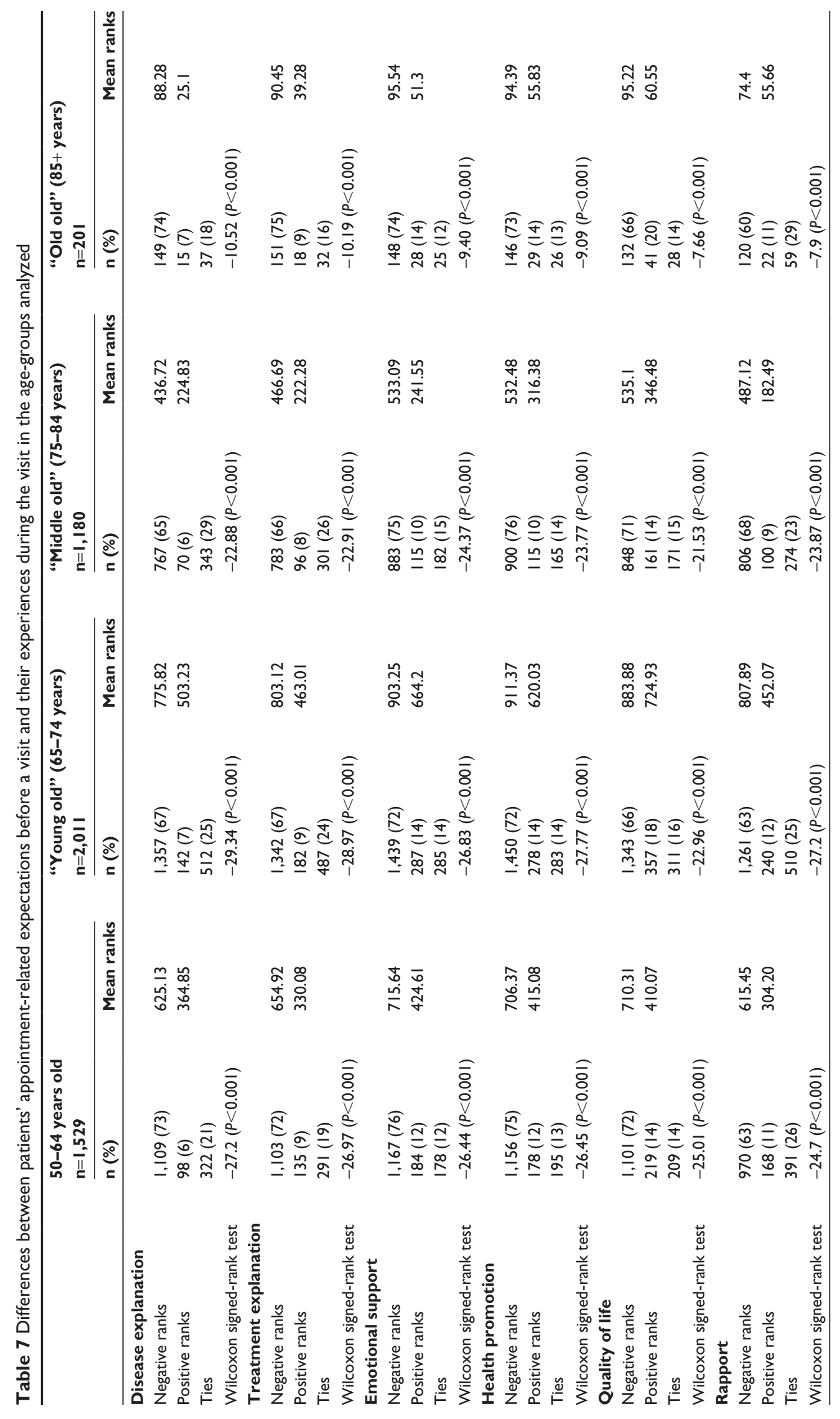


Table 8 Differences in patients' expectations for each age-group analyzed

\begin{tabular}{|c|c|c|c|c|c|c|c|c|}
\hline & \multicolumn{2}{|c|}{$\begin{array}{l}50-64 \text { years old } \\
n=I, 529\end{array}$} & \multicolumn{2}{|c|}{$\begin{array}{l}\text { “Young old" } \\
\text { (65-74 years) } \\
n=2,01 \text { I }\end{array}$} & \multicolumn{2}{|c|}{$\begin{array}{l}\text { “Middle old" } \\
\text { (75-84 years) } \\
n=I, 180\end{array}$} & \multicolumn{2}{|c|}{$\begin{array}{l}\text { "Old old" } \\
(85+\text { years }) \\
(\mathrm{n}=20 \mathrm{I})\end{array}$} \\
\hline & Mean & SD & Mean & SD & Mean & SD & Mean & SD \\
\hline \multicolumn{9}{|l|}{ Difference in disease } \\
\hline explanation & -1.33 & 1.47 & -0.99 & 1.23 & -0.96 & 1.15 & -1.23 & 1.19 \\
\hline \multirow[t]{3}{*}{ Dunn's test* } & \multicolumn{8}{|c|}{$50-64>65-74$ years, $Z=-6.64(P=0.001)$} \\
\hline & \multicolumn{8}{|c|}{$50-64>75-84$ years, $Z=-6.64(P=0.00 \mathrm{I})$} \\
\hline & \multicolumn{8}{|c|}{$85+>75-84$ years, $Z=2.66(P=0.047)$} \\
\hline \multicolumn{9}{|l|}{ explanation } \\
\hline \multirow[t]{2}{*}{ Dunn's test* } & \multicolumn{8}{|c|}{$50-64>65-74$ years, $Z=-7.05(P=0.001)$} \\
\hline & \multicolumn{8}{|c|}{$50-64>75-84$ years, $Z=-5.68(P=0.001)$} \\
\hline \multicolumn{9}{|l|}{$\begin{array}{l}\text { Difference in emotional } \\
\text { support }\end{array}$} \\
\hline \multirow[t]{3}{*}{ Dunn's test* } & \multicolumn{8}{|c|}{$50-64>75-84$ years, $Z=-4.04(P=0.00 \mathrm{I})$} \\
\hline & \multicolumn{8}{|c|}{$50-64>65-74$ years, $Z=-7.77(P=0.001)$} \\
\hline & \multicolumn{8}{|c|}{$75-84>65-74$ years, $Z=-2.93(P=0.021)$} \\
\hline Difference in health & -1.39 & 1.56 & -1.05 & 1.42 & -1.2 & 1.31 & -1.08 & 1.35 \\
\hline \multicolumn{9}{|l|}{ promotion } \\
\hline \multirow[t]{4}{*}{ Dunn's test* } & \multicolumn{8}{|c|}{$50-64>75-84$ years, $Z=-3.6 \mathrm{I}(P=0.00 \mathrm{I})$} \\
\hline & \multicolumn{8}{|c|}{$50-64>65-74$ years, $Z=-7.34(P=0.001)$} \\
\hline & \multicolumn{8}{|c|}{$50-64>85+$ years, $Z=-3.03(P=0.015)$} \\
\hline & \multicolumn{8}{|c|}{$75-85>65-74$ years, $Z=2.98(P=0.018)$} \\
\hline \multicolumn{9}{|l|}{$\begin{array}{l}\text { Difference in quality } \\
\text { of life }\end{array}$} \\
\hline \multirow[t]{2}{*}{ Dunn's test* } & \multicolumn{8}{|c|}{$50-64>65-74$ years, $Z=-7.1(P=0.00 \mathrm{I})$} \\
\hline & \multicolumn{8}{|c|}{$50-64>75-84$ years, $Z=-4.23(P=0.001)$} \\
\hline Difference in rapport & -0.95 & 1.2 & -0.83 & 1.15 & -0.98 & 1.07 & -0.67 & 1.08 \\
\hline \multirow[t]{3}{*}{ Dunn's test* } & \multicolumn{8}{|c|}{$50-64>85+$ years, $Z=-3.05(P=0.0 \mid 4)$} \\
\hline & \multicolumn{8}{|c|}{$75-85>65-74$ years, $Z=3.7(P=0.00 I)$} \\
\hline & $75-84=$ & ars, $Z=$ & $=0.002)$ & & & & & \\
\hline
\end{tabular}

Note: *In all comparisons, discrepancies between previsit expectations and experiences evaluated after the visit (absolute value) was greater or smaller.

aged $85+$ years before medical visits did not differ statistically from those of patients aged 50-64 years with regard to disease explanation, treatment explanation, and rapport. Arguably, patients aged $85+$ years had the same expectations toward these factors as the youngest participants. Therefore, it is important that doctors provide seniors with accurate information on diagnosis and treatment processes.

Evaluation of patients' appointment-related experiences evaluated after the visit demonstrated that all types of expectations differed among the age-groups in the study. The youngest patients (50-64 years) reported lower levels of disease and treatment explanation compared to those aged 65-74 and 75-84 years. Significantly, in the group aged 50-64 years, experiences related to emotional support, quality of life, health promotion, and rapport proved statistically lower than in the young-old, middle-old, and old-old groups. It is worth noting that patients aged $85+$ years rated doctorpatient rapport higher than all other groups, especially the group aged 75-84 years.
The youngest patients rated the doctor's behavior lowest on all but one of the dimensions. When it came to the rapport dimension, we observed a distinct pattern wherein the old-old group rated the doctor's behavior highest. Differences between age-groups were more pronounced when it came to experiences than previsit expectations. This was possibly not only due to differences in the perceptions of the various age-groups but could also be explained by changes in the doctor's behavior as he/she approaches people of different ages.

The relationship between the patient and the doctor, very important for patients aged $85+$ years, is a core element in building successful rapport, which largely depends on the patient's expectations and the ability of the doctor to satisfy them. It is conceivable that successful rapport can have a positive influence on seniors, in particular the oldest ones. This may have a positive effect on involvement in treatment, the health-promotion process, and expectations toward health and medical care. ${ }^{4,5}$ 
Our study showed that differences between previsit and postvisit measurements were statistically significant in all age-groups regarding all types of expectations. In the age-groups analyzed, patients who received less than they expected from doctors with respect to disease explanation, treatment explanation, emotional support, health promotion, quality of life, and rapport outnumbered those who received what they expected or more than they expected. Considering particular expectations, we are able to conclude that on disease and treatment explanation, the numbers of patients with negative assessments were higher in the youngest and old-old groups than in the other groups. As for quality of life, a higher number of negative evaluations was observed in the youngest and middle-old groups. When it comes to the remaining dimensions, the proportion of patients with negative assessments was similar across the age-groups. In the case of rapport, the number of those who received less than they expected was lower than for other types of expectations, which suggests that this expectation was less neglected by doctors than the others. Except for the rapport dimension, the differences were largest in the youngest group of patients, while the young-old group had the smallest discrepancies, ie, the highest and lowest levels of expectations within the same age-group. We could interpret this to mean that patients of preretirement age have high expectations that are not always satisfied, whereas those aged 65-74 years tend to have more realistic expectations and subsequently rate the doctor's behavior more in line with their expectations.

The pattern of differences between the age-groups in the experience categories varied slightly, though they seemed to be coherent. At the same time, the differences in the rapport category were the only ones that appeared to be significant among the youngest and the oldest participants, corresponding to similar differences found for previsit expectations. It should be noted that the old-old group had higher discrepancies on disease explanation than patients aged 75-84 years. This means that patients aged $85+$ years received less than they expected from doctors in comparison to the middle-old group. This observation was interesting, as Vieder et $\mathrm{al}^{28}$ observed that seniors tend to have high expectations toward medical visits, while being also more likely to return home with an abundance of confusing and conflicting information about their treatment. This may be caused by a failure to offer accurate information in nontechnical terms. Moreover, there may be cases where older patients feel confused and subsequently fail to seek clarification. A number of researchers have also found that among the most important patient expectations, we find disease and treatment explanation, stressing that patients and physicians ought to develop a relationship marked by open access to information. ${ }^{8,9,29}$ According to those authors, explanation of disease and treatment was strongly related to patients' level of satisfaction. Patients who are the most satisfied are more likely to adhere to treatment and self-care more actively. ${ }^{30,31}$

Our study showed that patients aged 75-84 years had a lower discrepancy in their expectation toward emotional support than patients aged 50-64 years, and had a higher discrepancy than patients aged 65-74 years. This could be explained by fact that older people expect their physicians to be friendly, kind, humorous, and patient during consultation..$^{32}$ In addition, there are positive correlations between patients' trust and their involvement in decisions about their health care, and this tends to increase with the patient's age..$^{33}$

The results confirmed that age is one of the sociodemographic variables that modifies patients' appointment-related expectations. This indicates that doctors should use an individual approach to provide accurate, balanced information about the treatment, while promoting realistic expectations and informed/shared decisions. In the same vein, the fulfillment of the patients' expectations is linked to the doctor's interpersonal skills. ${ }^{7,8}$ This underscores the significance of developing psychosocial competence among medical students. Such recommendations reflect the practical aspect of the data obtained from the PRACTA study.

In our study, we observed that regardless of the type of expectation, the largest discrepancies between previsit expectations and experiences evaluated after the visit were evident in the youngest group. Some studies suggest that patients get more positive experiences with age. ${ }^{34}$ However, Bowling and Rowe ${ }^{9}$ suggested that lower discrepancies between previsit expectations and experiences evaluated after the medical visit could be superficial. Perhaps this can be explained by a convergence between the previsit ideal and realism in patients' expectations, especially in the case of senior patients. This would at least partially support our finding that gaps between expectations and experiences were different for patients of preretirement age and those of older age. Fairhead and Rothwell ${ }^{35}$ and Lawler et al ${ }^{36}$ emphasize that there is increasing evidence of ageism in health care, and patients' seemingly positive experiences with growing age could be a result of this phenomenon. Accordingly, it is important to carry out a more detailed investigation into differences in expectations among patient age-groups.

It is worth mentioning that we also observed differences in educational levels among the different age-groups. 
With higher age, patients with primary education were more numerous, whereas the number of respondents with secondary or higher education decreased. The distribution of the respondents' place of residence was not balanced either, as patients who lived in large towns or cities were dominant in each age-group. Probably, differences in educational leveland not only age - could be an important factor influencing responses and attitudes to health care. However, this requires further empirical research.

The results of the PRACTA project provided interesting and complex data material regarding patients' expectations toward medical visits for different age-groups. The PRACTA project involved a large group of patients aged 50-98 years. Another strength of this study is the diversity of the patients' socioeconomic characteristics, which included factors like sex, marital status, education level, place of residence, work status, and financial situation. This allowed us to implement a detailed examination of the respondents. It should also be mentioned that the PRACTA project analyzed patients' expectations concerning not only various types of medical information (including information about causes of disease, treatment, health promotion, and quality of life) but also factors like emotional support and rapport. In the literature, it is emphasized that the most frequently analyzed patient expectations are related to information about disease and treatment, emotional support, rapport, quality of life, and health promotion. ${ }^{7,8,18-26}$ Nevertheless, there has so far been no study attempting to analyze these expectations together. The current study represents a comprehensive and unique attempt to do just that.

A strength of this study is also reflected in the methodology used, which allowed for a comprehensive analysis of patients' expectations in different age-groups. Patients completed questionnaires before and after an appointment with the doctor. Subsequently, we not only analyzed the patients' appointment-related expectations before the medical visit but also how they evaluated their experiences after the visit.

It is worth recognizing the limitations of the presented study: the study group consisted of purposefully selected seniors, a lack of earlier research on the topic, and singlemeasurement variables, which excluded the possibility of analyzing change over time. Despite these limitations, the results of the PRACTA project provided important information that could lead to a better understanding of seniors' expectations toward medical care.

\section{Conclusion}

The PRACTA study revealed differences related to age when it came to patients' expectations toward several factors, including disease explanation, treatment explanation, quality of life, rapport, and emotional support. Differences were not observed with regard to health promotion. Furthermore, an evaluation of patients' appointment-related experiences after a visit showed that that there were significant differences between age-groups concerning all types of expectations included in the study. Our study showed that differences between previsit and postvisit measurements were statistically significant with respect to all types of expectations in all the age-groups. In the groups analyzed, patients who received less than they expected from doctors regarding disease explanation, treatment explanation, emotional support, promotion, quality of life, and rapport outnumbered those who received what they expected or more than they had expected.

It is important that doctors share accurate and balanced information about treatment with patients, enabling realistic expectations and informed decisions. This is particularly reflected in how patients evaluate their experiences after a visit. Discrepancies between previsit expectations and experiences evaluated after the visit may negatively influence patients' attitude toward their own medical treatment.

\section{Disclosure}

The authors report no conflicts of interest in this work.

\section{References}

1. World Health Organization. Global Health and Aging. Geneva: WHO; 2011.

2. Oxley H. Policies for healthy ageing: an overview. 2009. Available from: http://www.oecd.org/officialdocuments/publicdisplaydocument pdf/?cote=DELSA/HEA/WD/HWP\%282009\%291\&docLanguage=En . Accessed May 17, 2017.

3. Wan H, Goodkind D, Kowal P. An Aging World: 2015. Suitland (MD): US Census Bureau; 2016.

4. Bowling A, Dieppe P. What is successful ageing and who should define it? BMJ. 2005;331:1548-1551.

5. Bowling A. Perceptions of active ageing in Britain: divergences between minority ethnic and whole population samples. Age Ageing. 2009;38:703-710.

6. Berhane A, Enquselassie F. Patient expectations and their satisfaction in the context of public hospitals. Patient Prefer Adherence. 2016;10: 1919-1928.

7. Bowling A, Rowe G, Lambert N, et al. The measurement of patients' expectations for health care: a review and psychometric testing of a measure of patients' expectations. Health Technol Assess. 2012;16: $1-509$.

8. Bowling A, Rowe G, McKee M. Patients' experiences of their healthcare in relation to their expectations and satisfaction: a population survey. J R Soc Med. 2013;106:143-149.

9. Bowling A, Rowe G. Psychometric properties of the new Patients' Expectations Questionnaire. Patient Exp J. 2014;1:111-130.

10. Rao JK, Weinberger M, Kroenke K. Visit-specific expectations and patient-centered outcomes: a literature review. Arch Fam Med. 2000;9: $1148-1155$.

11. Faller H, Vogel H, Bosch B. [Patient expectations regarding methods and outcomes of their rehabilitation: a controlled study of back pain and cancer patients]. Rehabilitation (Stuttg). 2000;39:205-214. German. 
12. Janzen JA, Silvius J, Jacobs S, Slaughter S, Dalziel W, Drummond N. What is a health expectation? Development of a pragmatic conceptual model from psychological theory. Health Expect. 2006;9:37-48.

13. Dawn AG, Lee PP. Patient expectations for medical and surgical care: a review of the literature and applications to ophthalmology. Surv Ophthalmol. 2004;49:513-524.

14. Sebo P, Herrmann FR, Bovier P, Haller DM. What are patients' expectations about the organization of their primary care physicians' practices? BMC Health Serv Res. 2015;15:328.

15. Jung HP, Wensing M, Olesen F, Grol R. Comparison of patients' and general practitioners' evaluations of general practice care. Qual Saf Health Care. 2002;11:315-319.

16. Horne R, Chapman SC, Parham R, Freemantle N, Forbes A, Cooper V. Understanding patients' adherence-related beliefs about medicines prescribed for long-term conditions: a meta-analytic review of the necessity-concerns framework. PLoS One. 2013;8:e80633.

17. Campbell JL, Ramsay J, Green J. Age, gender, socioeconomic, and ethnic differences in patients' assessments of primary health care. Qual Health Care. 2001;10:90-95.

18. Appleby J. How satisfied are we with the NHS? BMJ. 2011;342:d1836.

19. Bower P, King M, Nazareth I, Lampe F, Sibbald B. Patient preferences in randomised controlled trials: conceptual framework and implications for research. Soc Sci Med. 2004;61:685-695.

20. Dolovich L, Nair K, Sellors C, Lohfeld L, Lee A, Levine M. Do patients' expectations influence their use of medications? Qualitative study. Can Fam Physician. 2008;54:384-393.

21. Kravitz RL. Measuring patients' expectations and requests. Ann Intern Med. 2001;1:881-888

22. Kemicer-Chmielewska E, Rotter I, Pierzak-Sominka J, et al. Geriatrics patients' reasons to visit GPs. J Public Health Nurs Med Rescue. 2014;3:30-35.

23. Bakić-Mirić NM, Bakić NM. Successful doctor-patient communication and rapport building as the key skills of medical practice. Facta Univ Ser Med Biol. 2008;15:74-79.

24. Coulter A. What do patients and the public want from primary care? BMJ. 2005;331:1199-1201.
25. Leach CJ, Mandy A, Hankins M, et al. Patients' expectations of private osteopathic care in the UK: a national survey of patients. $B M C$ Complement Altern Med. 2013;13:122.

26. Kaptchuk TJ, Shaw J, Kerr CE, et al. "Maybe I made up the whole thing": placebos and patients' experiences in a randomized controlled trial. Cult Med Psychiatry. 2009;33:382-411.

27. Strzelecka AK, Nowak-Starz G, Karakiewicz B. Expectations of patients towards a primary care physician, related to the reason for the current visit, with particular emphasis on patients aged 65+. Fam Med Prim Care Rev. 2015;17:215-218.

28. Vieder JN, Krafchick MA, Kovach AC, Galluzzi KE. Physicianpatient interaction: what do elders want? J Am Osteopath Assoc. 2002; 102:73-78.

29. Mishra PH, Mishra T. Study of patient satisfaction at a super specialty tertiary care hospital. Indian J Clin Pract. 2014;25:624-634.

30. Asadi-Lari M, Tamburini M, Gray D. Patients' needs, satisfaction, and health related quality of life: towards a comprehensive model. Health Qual Life Outcomes. 2004;2:32.

31. Dwamena F, Holmes-Rovner M, Gaulden CM, et al. Interventions for providers to promote a patient-centred approach in clinical consultations. Cochrane Database Syst Rev. 2012;12:CD003267.

32. Marcinowicz L, Pawlikowska T, Oleszczyk M. What do older people value when they visit their general practitioner? A qualitative study. Eur J Ageing. 2014;11:361-367.

33 Butterworth JE, Campbell JL. Older patients and their GPs: shared decision making in enhancing trust. Br J Gen Pract. 2014;64:e709-e718.

34. Lyratzopoulos G, Elliott M, Barbiere JM, et al. Understanding ethnic and other socio-demographic differences in patient experience of primary care: evidence from the English General Practice Patient Survey. BMJ Qual Saf. 2012;21:21-29.

35. Fairhead JF, Rothwell PM. Underinvestigation and undertreatment of carotid disease in elderly patients with transient ischaemic attack and stroke: comparative population based study. BMJ. 2006; 333:525-527.

36. Lawler M, Selby P, Aapro MS, Duffy S. Ageism in cancer care. BMJ. 2014;348:g1614.
Patient Preference and Adherence

\section{Publish your work in this journal}

Patient Preference and Adherence is an international, peer-reviewed, open access journal that focuses on the growing importance of patient preference and adherence throughout the therapeutic continuum. Patient satisfaction, acceptability, quality of life, compliance, persistence and their role in developing new therapeutic modalities and compounds to optimize

\section{Dovepress}

clinical outcomes for existing disease states are major areas of interest for the journal. This journal has been accepted for indexing on PubMed Central. The manuscript management system is completely online and includes a very quick and fair peer-review system, which is all easy to use. Visit http://www. dovepress.com/testimonials.php to read real quotes from published authors. 